\title{
Factores de riesgo para presentar dolor crónico de tobillo en pacientes con fractura de tobillo clasificados con Danis Weber y AO en el Centenario Hospital Miguel Hidalgo
}

\author{
Chronic ankle pain risk factors in patients with \\ ankle fracture classified with Danis Weber and AO \\ at the Centenario Hospital Miguel Hidalgo
}

\author{
Ávila-Méndez, Christian M.; Ortiz-Díaz, Luis Gabriel; Flores- \\ Álvarez, Efrén
}

\author{
Christian M. Ávila-Méndez \\ Centenario Hospital Miguel Hidalgo. \\ Aguascalientes, Ags. México., México \\ Luis Gabriel Ortiz-Díaz avimen.cm@gmail.com \\ Centenario Hospital Miguel Hidalgo, \\ Aguascalientes, Ags. México., México \\ Efrén Flores-Álvarez \\ Centenario Hospital Miguel Hidalgo, \\ Aguascalientes, Ags. México, México
}

\author{
Lux Médica \\ Universidad Autónoma de Aguascalientes, México \\ ISSN: 2007-1655 \\ Periodicidad: Cuatrimestral \\ vol. 16, núm. 48,2021 \\ luxmedica.editorial@gmail.com
}

Recepción: 28/12/2020

Aprobación: 07/06/2021

URL:

https://revistas.uaa.mx/index.php/luxmedica/article/view/3322

Autor de correspondencia: avimen.cm@gmail.com

Poítica de acceso abierto La Revista Lux Médica proporciona un acceso abierto a su contenido, basado en el principio de que ofrecer un acceso libre a las investigaciones ayuda a incrementar el intercambio global del conocimiento. La LM no cobra ni cobrará ningún cargo a sus lectores por concepto de suscripción, ni a los autores por enviar, procesar o publicar sus artículos. Como condición de publicación, los autores acuerdan liberar sus derechos de autor bajo una licencia compartida, específicamente la licencia de Creative Commons Reconocimiento-NoComercial-Compartir Igual 4.0 Internacional Esta licencia permite a cualquier persona compartir, copiar y redistribuir el material en cualquier medio o formato bajo los siguientes términos: - Dar crédito al autor del texto - No hace uso del material con propósitos comerciales - No transformar o modificar el material. Los autores ceden el derecho de la primera publicación a esta revista, pero conservarn sus derechos de autor,
Resumen: Introducción: Las fracturas de tobillo son las lesiones óseas más comunes en cualquier grupo de edad y, por lo general, son causadas por un mecanismo de baja energía. Una de las clasificaciones más usadas es la de Danis-Weber, la cual nos habla de la probabilidad de lesión sindesmal; sin embargo, no nos habla de las complicaciones o probabilidad de padecer dolor crónico. Objetivo: Identificar los factores de riesgo que pudieran presentar los pacientes con fractura de tobillo que puedan influenciar la presencia de dolor crónico de tobillo. Material y métodos: Se realizó una revisión sistemática de 142 expedientes clínicos electrónicos de pacientes con diagnóstico de fractura de tobillo clasificada con Danis-Weber, en el periodo de 2016 a 2019, que cumplieron con los criterios de inclusión. Resultados: Se obtuvo una muestra de 142 pacientes, se analizaron variables como Weber, tratamiento, medición de claro medial, dolor crónico, complicación y mecanismo de lesión. Se obtuvieron diferencias estadísticamente significativas en las variables de medición de claro medial ( $\mathrm{p} \leq 0.01)$; $\mathrm{y}$, en cuanto a dolor crónico, en donde es más probable que los pacientes con Weber $\mathrm{B}$ o $\mathrm{C}$ presenten dolor crónico $(p=0.02)$. Conclusiones: Hay factores de riesgo para padecer dolor crónico de tobillo como el padecer una fractura de tipo Weber B o C; existe una relación en la probabilidad de lesión del ligamento deltoideo con la clasificación de Danis-Weber.

Palabras clave: dolor crónico, fractura de tobillo, Danis-Weber.

Abstract: Introduction: Ankle fractures are the most common bone injuries in any age group and are caused generally by a low-energy mechanism. One of the most applied classifications is Danis-Weber, which tells us about the probability of syndesmal injury. Objective: To identify the risk factors that patients with ankle fractures may present that could influence the presence of chronic ankle pain. Material and methods: A systematic review of 142 electronic medical records of patients with ankle fracture diagnosis with Danis-Weber classification from 2016 to 2019. Results: 142 patients were evaluated considering variables like 
de tal forma que pueden realizar otros acuerdos contractuales independientes y adicionales para la distribución no exclusiva de la versión del artículo publicado en esta revista ( por ejemplo, incluirlo en un repositorio institucional o publicarlo en un libro) siempre que indiquen claramente que el trabajo se publicó por primera vez en ésta.

\section{(1) (2)}

Esta obra está bajo una Licencia Creative Commons AtribuciónNoComercial-CompartirIgual 4.0 Internacional.

Cómo citar este artículo: Ávila-Méndez, C. M., Ortiz-Díaz, L. G., Flores-Álvarez, E. Factores de riesgo para presentar dolor crónico de tobillo en pacientes con fractura de tobillo clasificados con Danis Weber y AO en el Centenario Hospital Miguel Hidalgo. Lux Médica, 16(48). Recuperado a partir de https://revistas.uaa.mx/index.php/lu $\mathrm{xmedica/article/view/3322}$
Weber, treatment, medial clearance measurement, chronic pain, complication, and injury mechanism were analyzed. Statistically significant differences were obtained in the medial clearance measurement variables $(\mathrm{p} \leq 0.01)$; patients with Weber $\mathrm{B}$ or $\mathrm{C}$ are more likely to have chronic pain $(p=0.02)$. Conclusions: There are risk factors for suffering from chronic ankle pain (present a Weber $\mathrm{B}$ or $\mathrm{C}$ type fracture). There is a relationship in the probability of injury to the deltoid ligament with the DanisWeber classification.

Keywords: Chronic pain, Ankle fracture, Danis-Weber.

\section{INTRODUCCIÓN}

La fractura de la articulación del tobillo es una de las fracturas más comúnmente vistas en todas las edades, sin distinción de sexo o raza; sin embargo, se ha observado que hasta un $75 \%$ predomina en una edad productiva. ${ }^{1,2}$ Su etiología es casi siempre por traumatismo indirecto de baja energía, ocasionado con frecuencia durante la práctica deportiva o en las actividades de la vida diaria. ${ }^{3}$

En Estados Unidos de América, cerca de 5 millones de pacientes que acuden al departamento de Urgencias es debido a algún tipo de lesión de tobillo, de las cuales el $85 \%$ son esguinces y tan solo el $15 \%$ son fracturas. De todas las fracturas del cuerpo, cerca del $9 \%$ son fracturas de tobillo, pero, tomando en cuenta únicamente las fracturas de miembros pélvicos, la cifra asciende al 36\%, por lo que esta fractura es la que más comúnmente atiende el Traumatólogo Ortopedista. Se estima que en EE. UU. la fractura de tobillo genera un costo anual de $\$ 10$ mil millones de dólares. ${ }^{3,4,5}$

Las fracturas de tobillo suelen tener una distribución similar entre hombres y mujeres; sin embargo, la distribución por edad es más frecuente en hombres jóvenes y mujeres de edad avanzada. Debido al envejecimiento de la población en los últimos 30 años, la incidencia de fracturas de tobillo en mujeres de edad avanzada, se ha triplicado. ${ }^{4,6}$

Por lo general, la etiología de la fractura será por un traumatismo indirecto de baja energía, el cual podrá producirse posterior a la realización de actividades deportivas, en caso de adultos jóvenes, o en actividades de la vida diaria que, a raíz de un accidente, ocasionen una inversión o eversión forzada del tobillo. ${ }^{1}$

Es necesario realizar una adecuada y acertada identificación de la fractura, así como de las lesiones que esta haya causado en tejidos blandos y/o ligamentos. Para esta identificación acertada y cuidadosa, será necesario realizar un interrogatorio completo del paciente (que incluya antecedentes médicos detallados, así como el mecanismo de lesión), un examen físico, un examen radiográfico apropiado y ofrecer opciones de tratamiento inicial. ${ }^{4,7}$

Como se menciona anteriormente, para hacer un diagnóstico preciso y, con ello, lograr una adecuada clasificación de la fractura, es necesario realizar una evaluación radiográfica. Para esto es recomendado valerse de las proyecciones anteroposterior, lateral y anteroposterior con rotación medial de $15-20^{\circ}$, que también es llamada proyección de mortaja, mediante la cual podemos ver 
de mejor manera el espacio articular alrededor del astrágalo y el espacio de la sindesmosis. Este espacio, al ser medido a un centímetro de la superficie articular, debe medir de cinco a seis milímetros en una situación normal. Con estas proyecciones podremos entonces, realizar una adecuada clasificación de la fractura y, con ello, tener una adecuada planeación prequirúrgica. ${ }^{1,8,9}$

Ya que se ha hecho una adecuada y acertada clasificación de la fractura y, por ende, una buena planeación prequirúrgica, el punto clave para un óptimo resultado será la restauración anatómica de las estructuras involucradas en la reconstrucción de la articulación tibio-peroneo-astragalina, ya que, al tratarse de una articulación, es primordial lograr una consolidación primaria. ${ }^{4,10,11,12}$

Una manera sencilla pero útil de clasificar las fracturas de tobillo es con la clasificación de Danis-Weber, ya que es una clasificación relativamente simple, útil y conocida mundialmente.

La clasificación tiene sus inicios en el año de 1949, cuando Danis la describe por primera vez. Sin embargo, en 1965, Weber realiza modificaciones a la clasificación inicial y la actualiza con un criterio anatómico-radiológico que consiste en la evaluación de la fractura dependiendo la altura a la cual se encuentra la pérdida de continuidad del peroné. (Figura 1) 13,14,15,16,17

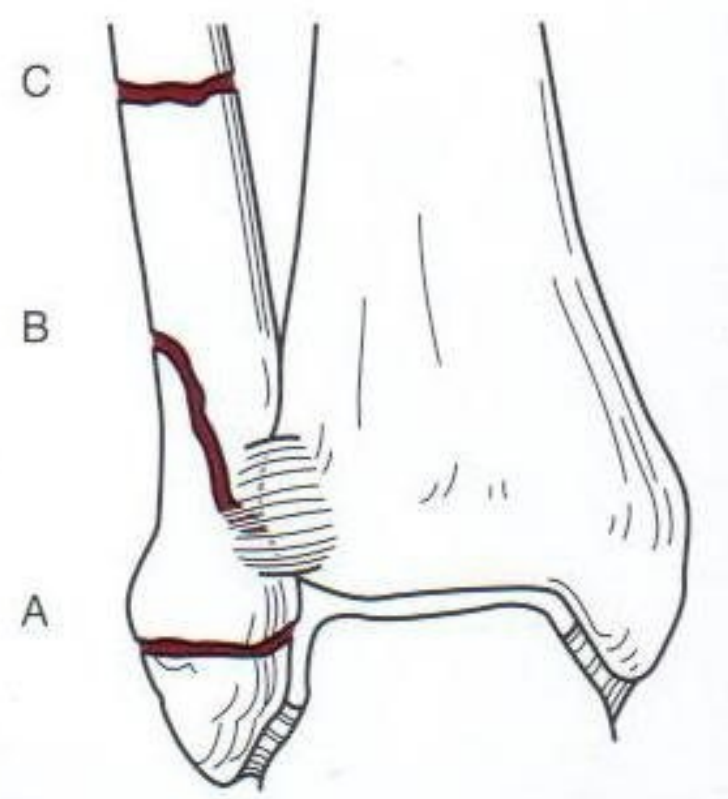

Figura 1.

Clasificación de Dannis-Weber. Fuente: Bucholz RW editor. Rockwood \& Green's. Fracturas en el adulto. Primera edición. Madrid: Marbán; 2003. ${ }^{18}$

\section{MATERIAL Y MÉTODOS}

Se realizó un estudio de casos y controles, retrospectivo, comparativo, analítico y descriptivo, en un periodo comprendido de 2016 al 2019 en el Centenario Hospital Miguel Hidalgo (CHMH) de Aguascalientes, Ags., México, en donde se analizaron variables como resultados radiográficos, pre-tratamiento, post- 
tratamiento, evolución a los 4.5 meses en conjunto con el tratamiento recibido y presencia de dolor crónico en pacientes con diagnóstico de fractura de tobillo que hayan sido clasificados utilizando la clasificación de Weber y la creada por la Arbeitsgemeinschaft für Osteosynthesefragen (AO).

Para la obtención de los expedientes de pacientes a revisar, se realizó una búsqueda en la base de datos del Centenario Hospital Miguel Hidalgo, de notas postquirúrgicas o diagnósticos iniciales o finales, así como de notas de valoración o interconsulta, que contuvieran las palabras: "Weber, fractura de tobillo, unimaleolar, bimaleolar, trimaleolar, Weber A, Weber B, Weber C”, en el periodo comprendido de 2016 a 2019.

Para la obtención de la bibliografía, se hizo una exploración en las plataformas de Pubmed, Science Direct y Scholar Google, en donde se buscaron artículos con las palabras clave: "Clasificación Danis Weber, fractura de tobillo, fracturas Weber, dolor crónico de tobillo, mediciones radiográficas en fractura de tobillo, epidemiología en México de fracturas de tobillo, lesión crónica de ligamento deltoideo, anatomía de tobillo".

En todos los casos se realizó planificación preoperatoria. Para confirmar el diagnóstico, se obtuvieron radiografías del tobillo afectado con vista anteroposterior y lateral. La selección de qué método quirúrgico se utilizó en el paciente se decidió por elección de cada médico tratante y dependiendo de los hallazgos intraoperatorios individualizados en cada caso.

El pre-tratamiento, post-tratamiento inmediato y post-retiro de tornillo situacional se analizó por el tipo de fractura de cada paciente, y se clasificó de acuerdo a los criterios de Danis-Weber y AO. Se midió el claro medial de acuerdo con un protocolo establecido a la distancia que se bisecó la dimensión vertical del maléolo medial; con una medida superior a $4 \mathrm{~mm}$ se consideró que había una lesión del ligamento deltoideo.

Criterios de inclusión. Pacientes de sexo indistinto, edad $\geq 14$ años al momento de la cirugía, con diagnóstico pre y postquirúrgico de fractura de tobillo que hayan sido clasificados utilizando la clasificación de Danis-Weber y AO y que se encontraran dentro del grupo clasificado como B o C, que contaran con expediente electrónico y radiográfico completo y disponible, así como con un mínimo de 4.5 meses de seguimiento postquirúrgico.

Criterios de exclusión. Pacientes con diagnóstico de fractura de tobillo que no cumplieran con la clasificación antes mencionada, pacientes con exposición de la fractura, expediente electrónico no disponible, expediente radiográfico no disponible, pacientes que no tuvieran un seguimiento mínimo de 4.5 meses posteriores a la reducción y fijación de la fractura.

Análisis estadístico. Para el análisis de los resultados se utilizó el programa SPSS 25 de la compañía IBM, realizando una estadística descriptiva con medidas de tendencia central y de dispersión paramétrica y no paramétrica, según el tipo de distribución de los datos. Para establecer las diferencias entre los grupos se utilizó la prueba de chi cuadrada o la prueba exacta de Fisher. Se consideró que había significancia estadística cuando el valor de p fue menor a 0.05 . Para medir la fuerza de asociación de las variables con el dolor crónico de tobillo, se estimó la razón de momios con intervalos de confianza de $95 \%$. 


\section{RESULTADOS}

Se encontró un total de 218 pacientes con diagnóstico de fractura de tobillo clasificada con Weber y AO de manera inicial en la nota de ingreso, de los cuales 45 pacientes fueron excluidos debido a cambio en el diagnóstico posterior a una segunda revisión y/o cambio de diagnóstico transquirúrgico, quedando un total de 173 pacientes.

Posteriormente, se excluyeron pacientes que no contaban con expediente radiográfico o clínico completo, y pacientes que no cumplían con un mínimo de 4.5 meses de evolución posterior al tratamiento recibido en el CHMH. Por lo que un total de 142 pacientes fue la muestra que cumplían con las características requeridas para el estudio.

De los 142 pacientes seleccionados, el $40.8 \%(n=58)$ fueron hombres y el $59.2 \%(\mathrm{n}=84)$ fueron mujeres (Figura 2), la media de edad fue de 39.46 años, con un rango de 14-80 años de edad. El lado más comúnmente afectado fue el derecho con una frecuencia de 75 pacientes (52.8\%), mientras que el lado izquierdo tuvo una frecuencia de 67 pacientes $(47.2 \%)$ afectados.

\section{Distribución por sexo}

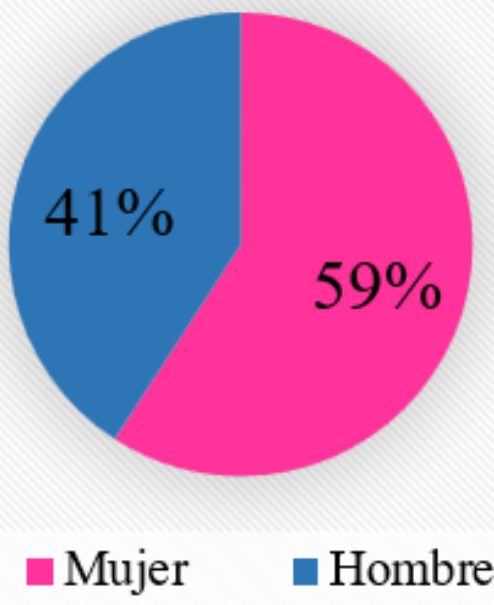

Figura 2.

Distribución por sexo

De la muestra estudiada, se observó que la clasificación de Dannis-Weber más común fue la de Fractura Unimaleolar A con una frecuencia de 35 pacientes (24.6\%), seguida de Fractura Bimaleolar B con una frecuencia de 28 pacientes (19.7\%) Tabla 1. 


\section{Tabla 1.}

Frecuencia de clasificación Danis-Weber.

Tabla 1. Frecuencia de clasificación Danis-Weber.

Frecuencia Porcentaje Porcentaje válido Porcentaje acumulado

\begin{tabular}{ccccc}
\hline Unimaleolar A & 35 & 24.6 & 24.6 & 24.6 \\
\hline Bimaleolar A & 3 & 2.1 & 2.1 & 26.8 \\
\hline Unimaleolar B & 27 & 19.0 & 19.0 & 45.8 \\
\hline Bimaleolar B & 28 & 19.7 & 19.7 & 65.5 \\
\hline Trimaleolar B & 12 & 8.5 & 8.5 & 73.9 \\
\hline Unimaleolar C & 15 & 10.6 & 10.6 & 84.5 \\
\hline Bimaleolar C & 20 & 14.1 & 14.1 & 98.6 \\
\hline Trimaleolar C & 2 & 1.4 & 1.4 & 100.0 \\
\hline Total & 142 & 100.0 & 100.0 & \\
\hline
\end{tabular}

Diagnóstico por Danis-Weber; n=142.

En los resultados, al hacer tablas cruzadas del nivel de fractura si era infrasindesmal o al nivel/suprasindesmal, se observó que la gran mayoría de los pacientes que tuvieron una fractura al nivel de la sindesmosis o por encima de ella, fueron sometidos a cirugía contra los que tuvieron una lesión infrasindesmal que recibieron tratamiento conservador, esto con un valor de $\mathrm{p}<0.001$.

Comparando el nivel de la fractura con la afectación del claro medial, se observó una estrecha relación del nivel de la fractura (al nivel o suprasindesmal) con la posibilidad de sufrir una afectación del ligamento deltoideo, obtenida mediante la medición del claro medial tanto en la valoración inicial, como en la medición post-tratamiento y la medición a los 4.5 meses de evolución, con un valor de $\mathrm{p} \leq 0.01$ (Figura 3). 


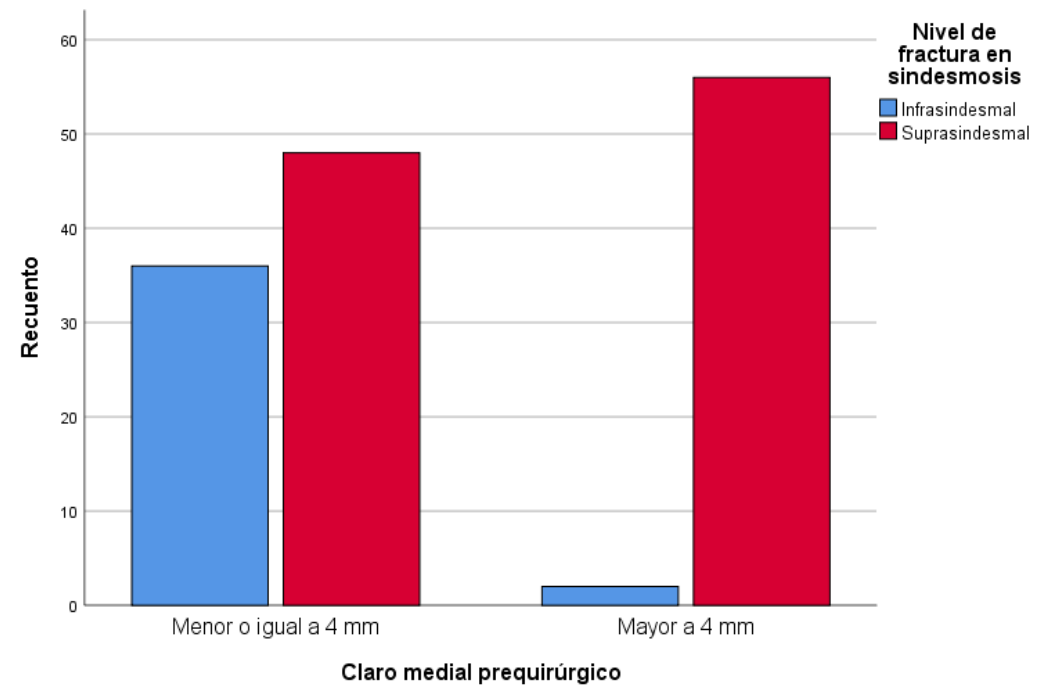

Figura 3

Nivel de fractura comparado con claro medial.

Revisando los casos de pacientes que padecieron dolor crónico y elaborando tabla cruzada con el nivel de fractura, se observó que los pacientes que padecen una fractura que se encuentre al nivel o por encima de la sindesmosis, son más propensos a padecer dolor crónico que aquellos que tuvieron una fractura infrasindesmal, con un valor de $\mathrm{p}=0.02$.

En cuanto a las complicaciones, se observó que es más probable que un paciente con una fractura que se encuentre al nivel de la sindesmosis o por encima de la misma, sufra alguna complicación, valor de $\mathrm{p}=0.008$. Las complicaciones observadas con mayor frecuencia fueron lesión de ligamento deltoideo, en 13 pacientes, seguida de pseudoartrosis aséptica y dehiscencia de herida, en seis y cinco casos, respectivamente, así como lesión crónica de sindesmosis, en cuatro pacientes (Tabla 2). Sin embargo, se encontró una baja incidencia de complicaciones con un 26.8\% $(n=38)$ de 142 casos. 
Tabla 2. Frecuencia de complicaciones por fractura.

\begin{tabular}{lrr}
\hline & Frecuencia & Porcentaje \\
\hline Ninguna & 104 & 73.2 \\
Fístula cutánea & 2 & 1.4 \\
Pseudoartrosis aséptica & 6 & 4.2 \\
Celulitis & 1 & .7 \\
Dehiscencia de herida & 5 & 3.5 \\
Pseudoartrosis séptica & 2 & 1.4 \\
Descobertura cutánea & 1 & .7 \\
Lesión crónica sindesmosis & 4 & 2.8 \\
Consolidación viciosa & 1 & .7 \\
Lesión crónica lig. deltoideo & 13 & 9.2 \\
Fuga de material & 2 & 1.4 \\
Fatiga tornillo transindesmal & 1 & .7 \\
Total & 142 & 100.0 \\
\hline
\end{tabular}

Datos expresados como frecuenciaa y porcentajes, $n=142$.

\section{Tabla 2}

Frecuencia de complicaciones por fractura.

\section{DISCUSIÓN}

En este estudio se realizó una evaluación de los casos y del manejo de pacientes con diagnóstico de fractura de tobillo clasificada como Danis-Weber y AO, las cuales fueron tratadas mediante tratamiento quirúrgico (en alguna de las tres modalidades) o conservador. Asimismo, se documentó a los pacientes que permanecen con dolor crónico post-tratamiento para analizar si existen factores de riesgo para padecer dolor crónico posterior a una fractura de tobillo.

La incidencia observada en este estudio en cuanto al género de los pacientes fue mayor de mujeres que hombres, con $59.2 \%$ de los casos, lo que es equiparable en comparación a la literatura internacional, como en el estudio realizado por Ryan C. Scheer et al, 2020, ${ }^{19}$ el cual reporta que en Estados Unidos la incidencia es de $56 \%$ en mujeres. Asimismo, la media de edad observada de 39.46 años, con un rango de 14-80 años, es similar a lo reportado en el mismo estudio.

Ryan C. Scheer y colaboradores, ${ }^{19}$ de igual manera en su estudio realizado en Estados Unidos, reportan que el mecanismo de lesión más común fue el de caída de su propio plano de sustentación, con un porcentaje de $35.68 \%$, seguido de actividades deportivas, con $35.26 \%$, lo cual, comparado con nuestro estudio sí es diferente, ya que en este estudio el mecanismo de lesión más frecuente fue 
el de caída del plano de sustentación, pero con un $66.9 \%$, seguido de caída de motocicleta y lesión por actividad deportiva, con $9.2 \%$ y $8.5 \%$, respectivamente.

En este estudio se observó que haciendo referencia a la necesidad de requerir tratamiento quirúrgico en relación al nivel de fractura, la gran mayoría, si no es que todas las fracturas tipo B y C de Weber (es decir al nivel de la sindesmosis o suprasindesmales), requirieron tratamiento quirúrgico, como lo que reporta el Dr. Tulio H. Makkozzay Pichardo ${ }^{1}$ en su estudio sobre complicaciones de las fracturas de tobillo.

El nivel de la fractura también guarda relación estrecha con la posibilidad de lesión del ligamento deltoideo, la cual se deduce de manera indirecta al observar un claro medial aumentado más de $4 \mathrm{~mm}$, así como lo reportado en el estudio hecho por Sous Sánchez y colaboradores, ${ }^{20}$ en donde la mayoría de los casos estudiados eran fracturas Weber B y C asociando comúnmente esta lesión a este tipo de fracturas.

Dentro de los hallazgos, se encontró en este estudio que los pacientes que padecen una fractura Weber $\mathrm{B}$ o $\mathrm{C}$ son más propensos a padecer dolor crónico, lo cual no está reportado en la literatura. Se buscó de manera intencionada si había una relación entre los pacientes que recibieron tratamiento quirúrgico y la presencia de dolor crónico; sin embargo, no se encontró por lo que descartamos el tratamiento quirúrgico como un factor de riesgo. Contrario a lo reportado por Hintermann et al., ${ }^{21}$ en este estudio no se encontró asociación entre la presencia de dolor crónico y lesión del ligamento deltoideo.

\section{CONCLUSIONES}

Con base en los resultados obtenidos del estudio, podemos concluir que hay factores de riesgo para padecer dolor crónico, como el padecer una fractura de tipo Weber B o C. Sin embargo, no se halló asociación entre una probable lesión del ligamento deltoideo y dolor crónico como se pudiera inferir. El estudio demostró que existe una relación en la probabilidad de lesión del ligamento deltoideo con la clasificación de Weber que los pacientes presenten. Por ende, es confiable planear el tratamiento que reciban con base en esta clasificación. Con este estudio pudimos comprobar más relación entre la clasificación de DanisWeber y la necesidad de requerir tratamiento quirúrgico, principalmente en los pacientes clasificados como B o C. Asimismo, encontramos que la clasificación de Danis-Weber guarda relación con la probabilidad de padecer lesión del ligamento deltoideo siendo más probable una fractura clasificada como Danis-Weber B o C, padezca algún tipo de complicación en comparación con las Weber A. En cuanto a las complicaciones, hubo una baja incidencia, por lo que se concluye que los tratamientos otorgados en el CHMH para los pacientes con fractura de tobillo son adecuados y cumplen los estándares en calidad de atención.

\section{BIBLIOGRAFÍA}

1. Makkozzay Pichardo, TH. Complicaciones de las fracturas de tobillo. Medigraphic Ortho-tips. 2006; 2(4): p.262-269. 2 
2. Jakub Olczak, Filip Emilson, Ali Razavian, Tone Antonsson, Andreas Stark \& Max Gordon. Ankle fracture classification using deep learning: automating detailed AO Foundation/Orthopedic Trauma Association (AO/OTA) 2018 malleolar fracture identification reaches a high degree of correct classification, Acta Orthopaedica, 2020; 91(1): p.102-108.DOI: 10.1080/17453674.2020.1837420

3. Yáñez Arauz JM, Arzac Ulla IR, Fiorentini G, Yáñez Arauz JM. Fracturas de tobillo. Comparación entre la cirugía abierta y el método mínimamente invasivo. Asociación Argentina de Ortopedia y Traumatología. 2016; 81(1): p 27-34.

4. Fonseca LLD, Nunes IG, Nogueira RR, Martins GEV, Mesencio AC, Kobata SI. Reproducibility of the Lauge-Hansen, Danis-Weber, and AO classifications for ankle fractures. Rev Bras Ortop. 2017;53(1):101-106. Published 2017 Dec 6. doi:10.1016/j.rboe.2017.11.013

5. Han SM, Wu TH, Wen JX, Wang Y, Cao L, Wu WJ, Gao BL. Radiographic analysis of adult ankle fractures using combined Danis-Weber and LaugeHansen classification systems. Sci Rep. 2020 May 6;10(1):7655. doi: 10.1038/ s41598-020-64479-2. PMID: 32376947; PMCID: PMC7203210.

6. Juto H, Nilsson H, Morberg P. Epidemiology of Adult Ankle Fractures: 1756 cases identified in Norrbotten County during 2009-2013 and classified according to AO/OTA. BMC Musculoskelet Disord. 2018; 19(1): p. 441-450.

7. Scheer RC, Newman JM, Zhou JJ, Oommen AJ, Naziri Q, Shah NV, Pascal SC, Penny GS, McKean JM, Tsai J, Uribe JA. Ankle Fracture Epidemiology in the United States: Patient-Related Trends and Mechanisms of Injury. The Journal of Foot \& Ankle Surgery. 2020; 59(3): p. 479-483.

8. Van Leeuwen CAT, Hoffman RPC, Donken CCMA, Van der Plaat LW, Schepers T, Hoogendoorn JM. The diagnosis and treatment of isolated type B fibular fractures: Results of a nationwide survey. Injury. 2019; 50(2): p. 579-589.

9. Lambert LA, Falconer L, Mason L. Ankle stability in ankle fracture. Journal of Clinical Orthopaedics and Trauma. 2020; 11(3): p. 375-379.

10. Schuberth JM, Collman DR, Rush SM, Ford LA. Deltoid Ligament Integrity in Lateral Malleolar Fractures: A Comparative Analysis of Arthroscopic and Radiographic Assessments. The Journal of Foot \& Ankle Surgery. 2004; 43(1): p. 20-29.

11. Schuberth JM, Jennings MM, Lau AC. Arthroscopy-Assisted Repair of Latent Syndesmotic Instability of the Ankle. Arthroscopy: The Journal of Arthroscopic and Related Surgery. 2008; 24(8): p. 868-874.

12. Zhao HM, Lu J, Zhang F, Wen XD, Li Y, Hao DJ, Liang XJ. Surgical treatment of ankle fracture with or without deltoid ligament repair: a comparative study. BMC Musculoskeletal Disorders. 2017; 18(1): p. 543-550.

13. Lampridis V, Gougoulias N, Sakellariou A. Stability in ankle fractures: diagnosis and treatment. EFORT Open Reviews. 2018; 3(5): p. 294-303.

14. Sous Sánchez JO, Navarro Navarro R, Navarro García R, Brito Ojeda E, Ruiz Caballero JA. Clasificación de las fracturas de tobillo. Canarias Médica y Quirúrgica. 2011; 9(25): p. 49-53.

15. Michelson JD. Fracturas del tobillo por rotación. Journal of the American Academy of Orthopaedic Surgeons (Edición Española). 2004; 3(1): p. 31-40.

16. Michel P J Van den Bekerom MPJ, Lamme B, Hogervorst M, Bolhuis HW. Which Ankle Fractures Require Syndesmotic Stabilization? The Journal of Foot and Ankle Surgery. 2007; 46(6): p. 456-463. 
17. Delaney JP, Charlson MD, Michelson JD. Ankle Fracture Stability-Based Classification: A Study of Reproducibility and Clinical Prognostic Ability. Journal of Orthopaedic Trauma. 2019; 33(9): p. 465-471.

18. Bucholz RW editor. Rockwood \& Green's. Fracturas en el adulto. Primera edición. Madrid: Marbán; 2003

19. Scheer RC et al. Ankle Fracture Epidemiology in the United States: Patient-Related Trends and Mechanisms of Injury. The Journal of Foot \& Ankle Surgery. 2020; 59(3): p. 479-483.

20. Sous Sánchez JO, Ruiz Caballero JA, Brito Ojeda ME, Navarro García R, Navarro Valdivielso ME, Navarro Navarro R. Fracturas de tobillo en deportistas. Estudio epidemiológico. Revista Internacional de Medicina y Ciencias de la Actividad Física y del Deporte. 2013; 13(50): p. 257-278.

21. Hintermann B, Boss A, Schäfer D. Arthroscopic Findings in Patients with Chronic Ankle Instability. Am J Sports Med. 2002 May-Jun;30(3): p. 402-9. 